Proyecciones Journal of Mathematics

Vol. 29, No 2, pp. 137-144, August 2010.

Universidad Católica del Norte

Antofagasta - Chile

\title{
POINTWISE BOUNDEDNESS AND EQUICONTINUITY IN $\beta$-DUALS
}

\author{
CHARLES SWARTZ \\ NEW MEXICO STATE UNIVERSITY, U. S. A. \\ Received: June 2010. Accepted: July 2010
}

\begin{abstract}
Let $E$ be a vector valued sequence space with operator valued $\beta$ dual $E^{\beta Y}$. If $E$ satisfies certain gliding hump assumptions, we show that pointwise bounded subsets of $E^{\beta Y}$ are sequentially equicontinuous. The result is established by considering uniform convergence of the elements in $E^{\beta Y}$.
\end{abstract}


There are two possibilities for the extension of the classical Uniform Boundedness Principle from Banach spaces to topological vector spaces. The classical Uniform Boundedness Principle for Banach spaces asserts that any pointwise bounded family of continuous linear operators between Banach spaces is uniformly bounded on bounded subsets of the domain space or, equivalently, is equicontinuous. This suggests two possible extensions of the Uniform Boundedness Principle to topological vector spaces; either find sufficient conditions for a pointwise bounded family of continuous linear operators between topological vector spaces to be uniformly bounded on families of bounded sets of the domain space or sufficient conditions for the family to be equicontinuous. Both of these possibilities have been addressed in the literature; see [Sw1], Chapter 4, for results of the former case and [Bo], III.6.2, [Ro], 2.2.1, [Wi],9.3.4, for results in the latter case. Similar possibilities exist for subsets of $\beta$-duals of vector valued sequence spaces. For example, the references [Sw1],12.5.7, [SS], Corollary 21, give sufficient conditions for a pointwise bounded subset of a $\beta$-dual to be uniformly bounded on bounded subsets of the sequence space. In this paper we address the second possibility above and consider sufficient conditions in the form of gliding hump properties for a pointwise bounded subset of the $\beta$-dual of a vector valued sequence space to be sequentially equicontinuous.

We begin by fixing the notation and terminology. Let $X, Y$ be Hausdorff topological vector spaces and let $E$ be a vector space of $X$ valued sequences containing the space $c_{00}(X)$ of all $X$ valued sequences which are eventually 0 . If $x$ is a sequence, we denote the $j^{\text {th }}$ coordinate of $x$ by $x_{j}$ so $x=\left\{x_{j}\right\}$ and if $z \in X$ and $j \in \mathbf{N}, e^{j} \otimes z$ denotes the sequence with $z$ in the $j^{\text {th }}$ coordinate and 0 in the other coordinates. The $\beta$-dual of $E$ with respect to $Y, E^{\beta Y}$, is defined to be

$$
E^{\beta Y}=\left\{\left\{T_{j}\right\} \subset L(X, Y): \sum_{j=1}^{\infty} T_{j} x_{j} \text { converges for every } x=\left\{x_{j}\right\} \in E\right\},
$$

where $L(X, Y)$ is the space of continuous linear operators from $X$ into $Y$. If $T=\left\{T_{j}\right\} \in E^{\beta Y}$ and $x=\left\{x_{j}\right\} \in E$, we write $T \cdot x=\sum_{j=1}^{\infty} T_{j} x_{j}$.

We now describe 2 gliding hump properties which will be used in the sequel. These gliding hump properties are used in sequence spaces in place of topological properties such as completeness and barrelledness in treating uniform boundedness or equicontinuity properties in topological vector spaces. An interval in $\mathbf{N}$ is a set of the form $[m, n]=\{j \in \mathbf{N}: m \leq j \leq n\}$, where $m \leq n$, and a sequence of intervals, $\left\{I_{j}\right\}$, is increasing if $\max I_{j}<$ $\min I_{j+1}$. If $I \subset \mathbf{N}, \chi_{I}$ will denote the characteristic function of $I$ and 
if $x=\left\{x_{j}\right\}$ is any scalar or vector valued sequence, $\chi_{I} x$ will denote the coordinatewise product of $\chi_{I}$ and $x$.

Definition 1. The space $E$ has the infinite gliding hump property ( $\infty$ GHP) if whenever $x \in E$ and $\left\{I_{j}\right\}$ is an increasing sequence of intervals, there exist a subsequence $\left\{N_{j}\right\}$ and $a_{N_{j}}>0, a_{N_{j}} \rightarrow \infty$ such that every subsequence of $\left\{N_{j}\right\}$ has a further subsequence $\left\{p_{j}\right\}$ such that the coordinate sum of the series $\sum_{j=1}^{\infty} a_{p_{j}} \chi_{I_{p_{j}}} x$ belongs to $E$.

The $\infty-G H P$ was introduced in [Sw4] to treat Orlicz-Pettis Theorems for multiplier convergent series with respect to the strong topology.

Examples of spaces with $\infty$-GHP are given in Appendices $\mathrm{B}$ and $\mathrm{C}$ of [Sw6]. For example, if $X$ is normed, then $c_{0}(X)$ and $l^{p}(X)(0<p<\infty)$ and any Banach AK-space have $\infty$-GHP.

A vector valued sequence space $E$ is a K-space if $E$ has a vector topology such that the coordinate maps $x=\left\{x_{j}\right\} \rightarrow x_{j}$ from $E$ to $X$ are continuous for every $j$.

Definition 2. The K-space $E$ has the zero gliding hump property (0-GHP) if for every null sequence $\left\{x^{j}\right\}$ in $E$ and every increasing sequence of intervals $\left\{I_{j}\right\}$, there is a subsequence $\left\{n_{j}\right\}$ such that the coordinate sum of the series $\sum_{j=1}^{\infty} \chi_{I_{n_{j}}} x^{n_{j}} \in E$.

The notion of the 0-GHP was introduced by Lee Peng Yee in [LPY].

Examples of K-spaces with 0-GHP are given in Appendices B and $\mathrm{C}$ of [Sw6]. For example, if $X$ is normed, then $c_{0}(X)$ and $l^{p}(X)(0<p \leq \infty)$ have 0-GHP.

Henceforth, we assume that $E$ is a K-space.

We now proceed to consider pointwise bounded and equicontinuous subsets of $E^{\beta Y}$. As in [Sw7], we do this by considering uniform convergence of elements in $E^{\beta Y}$. We first require a lemma.

Lemma 3. Let $A \subset E^{\beta Y}$ and $x^{k} \rightarrow 0$ in $E$. Assume that

(*) for every $x \in E$, the series $\sum_{j=1}^{\infty} T_{j} x_{j}$ converge uniformly for $T \in A$.

If the series $\sum_{j=1}^{\infty} T_{j} x_{j}^{k}$ do not converge uniformly for $T \in A, k \in \mathbf{N}$, there exist a symmetric neighborhood of $0, V$, in $Y, T^{k} \in A$, a subsequence $\left\{n_{k}\right\}$ and an increasing sequence of intervals $\left\{I_{k}\right\}$ such that $\sum_{l \in I_{k}} T_{l}^{k} x_{l}^{n_{k}} \notin V$. 
Proof : If the conclusion fails, there exists a symmetric neighborhood $U$ of 0 in $Y$ such that for every $k$ there exist $p_{k}>k, T^{k} \in A, x^{n_{k}}$ such that $\sum_{l=p_{k}}^{\infty} T_{l}^{k} x_{l}^{n_{k}} \notin U$. Pick a symmetric neighborhood $V$ of 0 in $Y$ such that $V+V \subset U$. For the condition above with $k=1$, there exist $p_{1}>1, T^{1} \in A, n_{1}$ such that $\sum_{l=p_{1}}^{\infty} T_{l}^{1} x_{l}^{n_{1}} \notin U$. Pick $q_{1}>p_{1}$ such that $\sum_{l=q_{1}+1}^{\infty} T_{l}^{1} x_{l}^{n_{1}} \in V$ so that $\sum_{l=p_{1}}^{q_{1}} T_{l}^{1} x_{l}^{n_{1}} \notin V$. By $\left(^{*}\right)$ there exists $N_{1}>p_{1}$ such that $\sum_{l=p}^{q} T_{l} x_{l}^{r} \in V$ for $q>p>N_{1}, T \in A, 1 \leq r \leq n_{1}$. As above there exist $q_{2}>p_{2}>N_{1}, T^{2} \in A, n_{2}$ such that $\sum_{l=p_{2}}^{q_{2}} T_{l}^{2} x_{l}^{n_{2}} \notin V$. Note that $n_{2}>n_{1}$. Put $I_{1}=\left[p_{1}, q_{1}\right], I_{2}=\left[p_{2}, q_{2}\right]$ and continue.

Remark 4. Lemma 3 is analogous to 2.15 of [Sw6] or Lemma 2 of [Sw5]. Condition $\left(^{*}\right)$ is used in order to construct the subsequence $\left\{n_{k}\right\}$ which is not necessary in these results. By 2.32 of [SW6], $\left(^{*}\right)$ holds if $A$ is pointwise bounded and $E$ has $\infty-G H P$.

To prove our result on the uniform convergence of pointwise bounded subsets of $\beta$-duals we use the Antosik-Mikusinski Matrix Theorem. We give a brief description of the version which will be used. Assume that $M=\left[m_{i j}\right]$ is an infinite matrix with values in $Y$ satisfying the following two conditions:

\section{(1) the columns of $M$ converge and}

(2) every increasing sequence of integers $\left\{\mathrm{n}_{j}\right\}$ has a further subsequence $\left\{m_{j}\right\}$ such that the series $\sum_{j=1}^{\infty} m_{i m_{j}}$ converge and $\lim _{i} \sum_{j=1}^{\infty} m_{i m_{j}}$ exists.

Such a matrix is called a $K$-matrix. One conclusion of the AntosikMikusinski Matrix Theorem asserts that the diagonal of $M, m_{i i}$, converges to 0 , and this is the version we use below. This version of the theorem and more general versions can be found in [Sw1], Chapter 2 or [Sw6], Appendix D.

Theorem 5. Assume that $E$ has $\infty-G H P$ and 0-GHP. If $A \subset E^{\beta Y}$ is pointwise bounded on $E$ and $x^{k} \rightarrow 0$ in $E$, then the series $\sum_{l=1}^{\infty} T_{l} x_{l}^{k}$ converge uniformly for $T \in A$ and $k \in \mathbf{N}$.

Proof : As noted in Remark 4 condition $\left(^{*}\right)$ is satisfied since $E$ has $\infty$-GHP. Suppose the conclusion fails and let the notation be as in Lemma 3. By 0 -GHP there exist a subsequence $\left\{r_{k}\right\}$ of $\left\{n_{k}\right\}$ and an increasing sequence of intervals $\left\{I_{k}\right\}$ such that $\sum_{k=1}^{\infty} \sum_{l \in I_{k}} e^{l} \otimes x_{l}^{r_{k}}=\sum_{k=1}^{\infty} \chi_{I_{k}} x^{r_{k}}=x \in E$. To avoid cumbersome notation later assume that $r_{k}=n_{k}$. Then

$$
\text { (\#) } \sum_{l \in I_{k}} T_{l}^{k} x_{l}^{n_{k}}=\sum_{l \in I_{k}} T_{l}^{k} x_{l} \notin V
$$


as in Lemma 3. Let the notation be as in the definition of $\infty$-GHP (Definition 1). Define a matrix

$$
M=\left[m_{i j}\right]=\left[\sum_{l \in I_{j}}\left(\frac{1}{a_{N_{i}}} T_{l}^{N_{i}}\right)\left(a_{N_{j}} x_{l}\right)\right] .
$$

We show that $M$ is a $K$-matrix. First, the columns of $M$ converge to 0 by the pointwise bounded assumption and the fact that $a_{N_{i}} \rightarrow \infty$ so (1) above holds. Next, for condition (2), given any subsequence of $\left\{N_{j}\right\}$ there exists a further subsequence $\left\{s_{j}\right\}$ such that

$$
\sum_{j=1}^{\infty} a_{s_{j}} \chi_{I_{s_{j}}} x=\sum_{j=1}^{\infty} a_{s_{j}} \sum_{l \in I_{s_{j}}} e^{l} \otimes x_{l}=y \in E .
$$

So

$$
\sum_{j=1}^{\infty} m_{i s_{j}}=\sum_{j=1}^{\infty} \sum_{l \in I_{s_{j}}}\left(\frac{1}{a_{N_{i}}} T_{l}^{N_{i}}\right)\left(a_{s_{j}} x_{l}\right)=\left(\frac{1}{a_{N_{i}}} T_{l}^{N_{i}}\right) \cdot(y) \rightarrow 0
$$

by pointwise boundedness and $a_{N_{i}} \rightarrow \infty$. By the Antosik-Mikusinski Matrix Theorem the diagonal of $M$ converges to 0 contradicting (\#).

The pair $(X, Y)$ has the sequential uniform boundedness property (SUB) if every pointwise bounded family $B \subset L(X, Y)$ is sequentially equicontinuous. For example, if $X$ is a complete metric linear space or a metrizable barrelled locally convex space, $(X, Y)$ has SUB (see [Ro]2.2.1 for the metric linear case and [Bo] III.6.2 or [Wi] 9.3.4 for the barrelled case).

Theorem 6. Assume that $A \subset E^{\beta Y}$ is pointwise bounded on $E, x^{k} \rightarrow 0$ in $E$ and $(X, Y)$ has the SUB property. If the series $\sum_{l=1}^{\infty} T_{l} x_{l}^{k}$ converge uniformly for $T \in A$ and $k \in \mathbf{N}$, then $\lim _{k} T \cdot x^{k}=0$ uniformly for $T \in A$.

Proof : Let $U$ be a neighborhood of 0 in $Y$ and pick a neighborhood of $0 V$ such that $V+V \subset U$. There exists $N$ such that $\sum_{l=N+1}^{\infty} T_{l} x_{l}^{k} \in V$ for $T \in A, k \in \mathbf{N}$. For $1 \leq l \leq N,\left\{T_{l}: T \in A\right\}$ is pointwise bounded on $X$ since $A$ is pointwise bounded and $T_{l} z=T \cdot\left(e^{l} \otimes z\right)$ for $z \in X$. Therefore, $\left\{T_{l}: T \in A\right\} \subset L(X, Y)$ is sequentially equicontinuous by the SUB property. Since $\lim _{k} x_{l}^{k}=0$ for each $l$, there exists $k_{0}$ such that $k \geq k_{0}$ implies $\sum_{l=1}^{N} T_{l} x_{l}^{k} \in V$. Hence, if $k \geq k_{0}$,

$$
T \cdot x^{k}=\sum_{l=1}^{N} T_{l} x_{l}^{k}+\sum_{l=N+1}^{\infty} T_{l} x_{l}^{k} \in V+V \subset U .
$$


From Theorems 5 and 6 , we have a sequential equicontinuity version of the Uniform Boundedness Theorem.

Theorem 7. Assume that $E$ has $\infty-G H P$ and $0-G H P$ and that $(X, Y)$ has the SUB property. If $A \subset E^{\beta Y}$ is pointwise bounded on $E$ and $x^{k} \rightarrow 0$ in $E$, then $T \cdot x^{k} \rightarrow 0$ uniformly for $T \in A$ (that is, $A$ is sequentially equicontinuous).

Without gliding hump assumptions the conclusion of Theorem 7 may fail.

Example 8. Let $c_{00}$, the space of all scalar sequences which are eventually 0 , have the sup-norm so $\left(c_{00}\right)^{\beta}=s$, the space of all sequences. Let $e$ be the sequence with 1 in each coordinate and let $e^{j}$ be the sequence with 1 in the $j^{\text {th }}$ coordinate and 0 in the other coordinates. The sequence $\left\{\sum_{j=1}^{k} e^{j} / k\right\}$ converges to 0 in $c_{00}$, but $e \cdot\left(\sum_{j=1}^{k} e^{j} / k\right)=1$ for each $k$. Therefore, $\{e\}$ is pointwise bounded on $c_{00}$, but is not sequentially equicontinuous.

Concerning the SUB assumption in Theorem 7, we have

Remark 9. If the conclusion of Theorem 7 is to hold, it is necessary that $(X, Y)$ has the $S U B$ property. For suppose $B \subset L(X, Y)$ is pointwise bounded and $z_{j} \rightarrow 0$ in $X$. Then $A=\{(T, 0,0, \ldots): T \in B\} \subset E^{\beta Y}$ is pointwise bounded on $E$ so if the conclusion of Theorem 7 holds, $\lim _{j} T z_{j}=$ $\lim _{j}(T, 0,0, \ldots) \cdot\left(e^{1} \otimes z_{j}\right)=0$ uniformly for $T \in B$.

Finally, note that sequential equicontinuity implies a strong uniform boundedness result.

Proposition 10. If $A \subset E^{\beta Y}$ is sequentially equicontinuous, then $A$ is uniformly bounded on bounded subsets of $E$.

Proof : Let $B \subset E$ be bounded and $t_{k} \rightarrow 0,\left\{T^{k}\right\} \subset A,\left\{x^{k}\right\} \subset B$. Then $t_{k} x^{k} \rightarrow 0$ in $E$ so $T^{k} \cdot\left(t_{k} x^{k}\right) \rightarrow 0$ and $A(B)$ is bounded.

The following example shows the converse of Proposition 10 is false and that the equicontinuity result of Theorem 7 is stronger than the uniform boundedness results of [Sw7]. Of course, the results in [Sw7] involve different gliding hump assumptions. 
Example 11. Consider $l^{2}$ with the weak topology $\sigma\left(l^{2}, l^{2}\right)$. The set $\left\{e^{j}\right\} \subset$ $l^{2}=\left(l^{2}\right)^{\beta}$ is uniformly bounded on $\sigma\left(l^{2}, l^{2}\right)\left(=\|\cdot\|_{2}\right)$ bounded subsets of $l^{2}$ but is not sequentially equicontinuous $\left(e^{j} \rightarrow 0\right.$ in $\sigma\left(l^{2}, l^{2}\right)$ but $e^{j} \cdot e^{j}=1$ for all $j$ ).

From Theorem 7 we have a uniform boundedness result.

Corollary 12. If the hypothesis of Theorem 7 are satisfied and $A \subset E^{\beta Y}$ is pointwise bounded, then $A$ is uniformly bounded on bounded subsets $B \subset E$.

A stronger uniform boundedness result is given in Theorem 12.5.7 of [Sw1].

\section{References}

[1] [Bo] N. Bourbaki, Espaces Vectoriels Topologiques, Livre V, Herman, Paris, (1976).

[2] [LPY] Lee Peng Yee, Sequence Spaces and the Gliding Hump Property, Southeast Asia Bull. Math., Special Issue, pp. 65-72, (1993).

[3] [LS] Li Ronglu and C. Swartz, Spaces for Which the Uniform Boundedness Principle Holds, Studia Sci. Math. Hung., 27, pp. 379-384, (1992).

[4] [Ro] S. Rolewicz, Metric Linear Spaces, Polish Sci. Publ., Warsaw, (1972).

[5] [SS] C. Stuart and C. Swartz, Uniform Convergence in the Dual of a Vector-Valued Sequence Space, Taiwan. J. Math., 7, pp. 665-676, (2003).

[6] [Sw1] C. Swartz, Infinite Matrices and the Gliding Hump, World Sci. Publ. Singapore, (1996).

[7] [Sw2] C. Swartz, A Multiplier Gliding Hump Property for Sequence Spaces, Proy. Revista de Mat., 20, pp. 20-32, (2001).

[8] [Sw3] C. Swartz, Uniform Boundedness in Vector-Valued Sequence Spaces, Proy. J. Math., 23, pp. 236-240, (2004). 
[9] [Sw4] C. Swartz, Orlicz-Pettis Theorems for Multiplier Convergent Operator-Valued Series, Proy. J. Math., 23, pp. 61-72, (2004).

[10] [Sw5] C. Swartz, Uniform Convergence of Multiplier Convergent Series, Proy. J. Math., 26, pp. 27-35, (2007).

[11] [Sw6] C. Swartz, Multiplier Convergent Series, World Sci. Publ., Singapore, (2009).

[12] [Sw7] C. Swartz, Boundedness and Uniform Convergence in $\beta$-Duals, Proy. J. Math., 29, pp. 77-84, (2010).

[13] [Wi] A. Wilansky, Modern Methods in Topological Vector Spaces, McGraw-Hill, NY, (1978).

\section{Charles Swartz}

Mathematics Department

New Mexico State University

Las Cruces, NM 88003,

U. S. A.

e-mail : cswartz@nmsu.edu 